\title{
Qualitätsmessung und Qualitätsmanagement in der Praxis - Verbesserung als Ziel
}

Viele Studien zeigen, dass die Qualität der Patientenversorgung uneinheitlich ist und damit nach wie vor Verbesserungspotenzial besteht $[1,2,3]$. Qualitätsmanagement kann daher wesentlich dazu beitragen, die Ergebnisse weiter zu verbessern $[4,5,6]$.

Voraussetzung ist eine zuverlässige Messung, die das Erkennen und das gezielte Angehen von möglichen Problemfeldern erlaubt. Auch neue Versorgungsformen, die mit dem Anspruch einer medizinischen Qualitätsverbesserung antreten, bedürfen diesbezüglich einer empirischen Überprüfung. Eine Steigerung der Anreize zur Qualitätsverbesserung verspricht man sich ferner von neuen Vergütungsformen, die messbare Qualitätselemente zumindest zur Modifikation einer Grundvergütung einbeziehen. Last but not least verlangt die Öffentlichkeit nach einer anbieterbezogenen Darstellung der medizinischen Qualität.

Alle diese Ansätze benötigen zuverlässige, mit vertretbarem Aufwand einsetzbare Messverfahren, die insbesondere auch eine Analyse der Behandlungsergebnisse erlauben. Es ist inzwischen nicht mehr die Frage, ob die für verschiedene administrative Zwecke erfassten medizinischen Daten für die Messung eingesetzt werden, sondern wie sie genutzt und weiterentwickelt werden können. Es zeichnet sich ferner ab, dass es für die Messung von Langzeitergebnissen außerhalb einiger Spezialbereiche und klinischer Studien kaum eine Alternative zur Verwendung der administrativen Daten geben wird, da eine umfangreiche, vom Ansatz her flächendeckende und hinreichend vollständige Nachverfolgung einer zunehmenden Zahl von definierten Erkrankungen oder Prozeduren mit anderen Mitteln (Erfassungsdaten) so aufwendig würde, dass sie in der Praxis nicht umsetzbar und auch nicht durchsetzbar wäre.

Der QMR Kongress 2011 stellt den aktuellen Stand der Messung mit Routinedaten dar. Neue Entwicklungen der Agency for Healthcare Research and Quality aus den USA, die Strategien in der Schweiz und Österreich, die jeweils aus den German Inpatient Quality Indicators [7] abgeleitete Systeme auf nationaler Ebene einsetzen, sowie die verschiedenen Entwicklungen in Deutschland in stationärer, ambulanter und sektorübergreifender Sicht werden von Entwicklern und Verantwortlichen aus erster Hand präsentiert. Die parallele Darstellung von konkreten Managementverfahren und dem Prozess des Peer Review, die ba- sierend auf den Messergebnissen die praktische Umsetzung von Verbesserungen im Alltag zum Ziel haben, spannt den Bogen zur Praxis.

Neue Versorgungsstrukturen wie Disease-Management-Programme bedürfen einer Überprüfung und auch eines Monitorings hinsichtlich ihrer tatsächlichen Wirkungen auf die medizinische Versorgungsqualität. Auch hierfür werden Analysen der Routinedaten verwendet und Qualitätsindikatoren benötigt. Daher berücksichtigen wir diesen Bereich erstmals auf unserer Konferenz. Das Verfahren Qualitätssicherung mit Routinedaten (QSR) [8] zeigt, dass die Qualitätsmessung nicht mehr nur eine interne Angelegenheit der Leistungserbringer ist, sondern zunehmend auch extern, beispielsweise seitens der Krankenkassen, durchgeführt, weiterentwickelt und im Versorgungsmanagement eingesetzt wird.

Die Tagung soll mit ihrem institutionsübergreifenden Ansatz dazu beitragen, den Meinungsaustausch zwischen Theoretikern und Praktikern der Beteiligten aller Bereiche zu fördern, um damit einen Beitrag zur Verbesserung der Instrumente für die Qualitätsmessung und damit letztlich zur Verbesserung der medizinischen Ergebnisse zu leisten.

Literatur

1 Peterson ED, Roe MT, Mulgund J, DeLong ER, Lytle BL, Brindis RG, Smith Jr SC, Pollack Jr CV, Newby $L K$, Harrington $R A$, Gibler WB, Ohman EM. Association Between Hospital Process Performance and Outcomes Among Patients With Acute Coronary Syndromes. JAMA 2006; 295: 1912-1920

2 Wöckel A, Kurzeder C, Geyer V, Novasphenny I, Wolters $R$, Wischnewsky M, Kreienberg R, Varga D. Effects of guideline adherence in primary breast cancer - a 5year multi-center cohort study of 3976 patients. Breast 2010; 19: 120-127

3 Kohn LT, Corrigan JM, Donnaldson MSEds . To err is human: Building a Safer Health System. Washington DC: Institut of Medicine: 1999

4 Buetow SA, Roland M. Clinical governance: bridging the gap between managerial and clinical approaches to quality of care. Quality in Health Care; 8: 184-190

5 Helios Kliniken. Ergebnisqualität sicher messen und aktiv verbessern - Erfahrungen. Medizinischer Jahresbericht der Helios Kliniken Gruppe 2006/2007. Berlin: Helios Kliniken GmbH: 2007

6 Rink O. Evaluation zeigt: Es gibt noch Verbesserungspotential. Dtsch Ärztebl 2011; 108: A 1518-1520

7 Mansky T, Nimptsch U, Winklmair C, Vogel K, Hellerhoff F. G-IQI | German Inpatient Quality Indicators Version 3.1. Berlin: Universitätsverlag der TU Berlin: 2007

8 AOK-Bundesverband, Forschungs- und Entwicklungsinstitut für das Sozial- und Gesundheitswesen SachsenAnhalt (FEISA), HELIOS Kliniken, Wissenschaftliches Institut der AOK (WIdO). Qualitätssicherung der stationären Versorgung mit Routinedaten (QSR) - Abschlussbericht Bonn: WIdO: 2007

\section{T. Mansky ${ }^{1}$ \\ J. Klauber ${ }^{2}$ \\ R. Kuhlen ${ }^{3}$ \\ P. Scriba 4}

Qualitätsmanagement

1 Technische Universität Berlin

2Wissenschaftliches Institut der AOK

3HELIOS Kliniken

4Initiative Qualitätsmedizin

Bibliografie

Dol 10.1055/s-0031-1286071 Dtsch Med Wochenschr 2011; 136:S41-S42 - (c) Georg Thieme Verlag KG Stuttgart · New York . ISSN 0012-0472

Korrespondenz Prof. Dr. Thomas Mansky Technische Universität Berlin Fachgebiet Strukturentwicklung und Qualitätsmanagement im Gesundheitswesen Straße des 17. Juni 135 10623 Berlin

Tel. 030/314-29805 eMail thomas.mansky@tu-berlin.de 

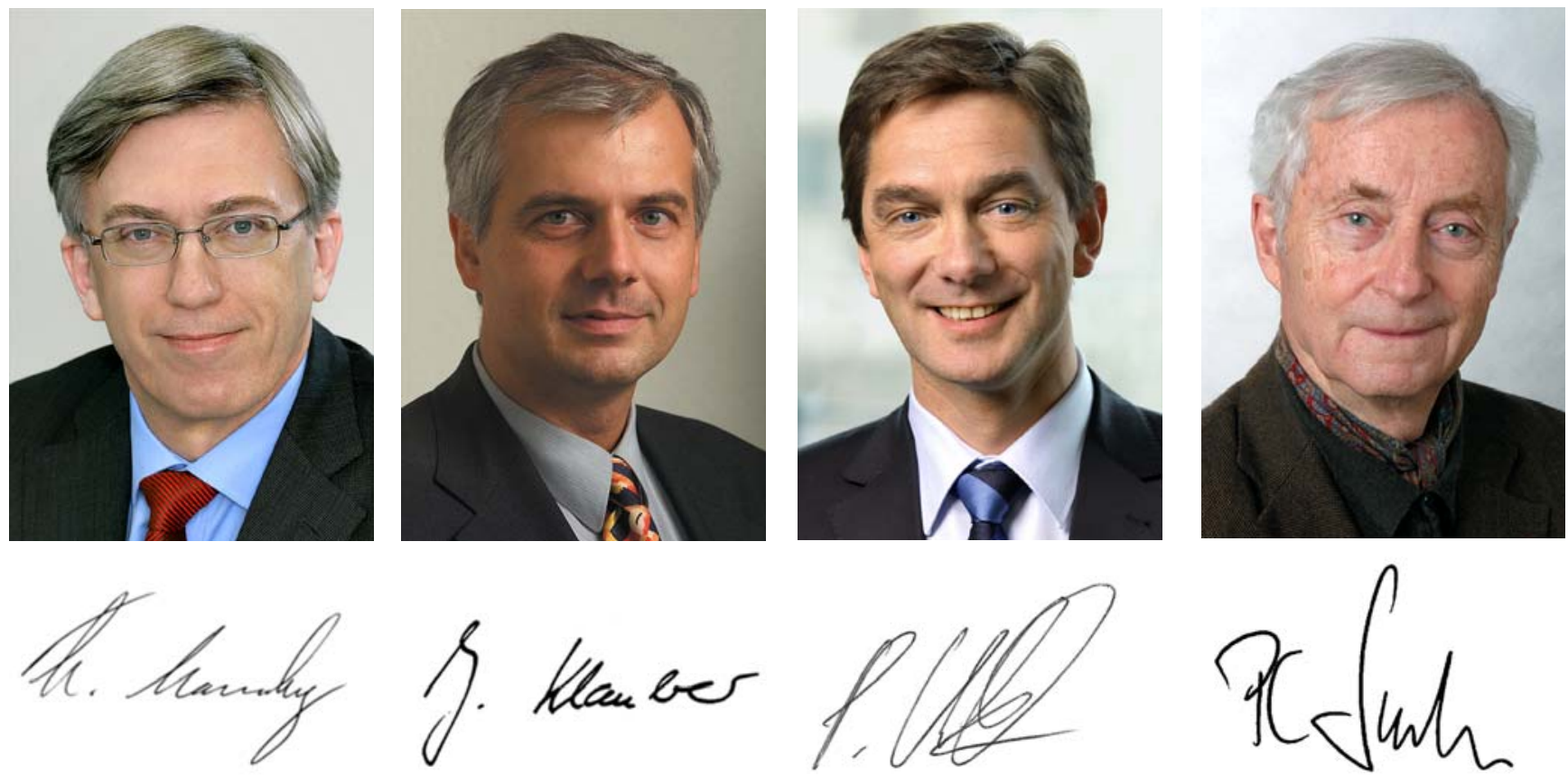

Prof. Dr. Thomas Mansky Technische Universität Berlin

Jürgen Klauber

Wissenschaftliches Institut der AOK
Prof. Dr. Ralf Kuhlen

HELIOS Kliniken

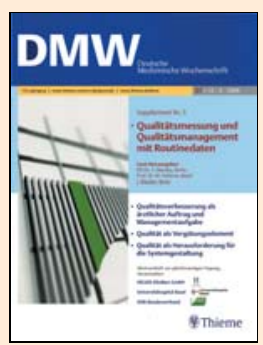

DMW S 5/2008

Qualitätsmessung und Qualitätsmanagement mit Routinedaten

Gast-Herausgeber:

PD Dr. T. Mansky, Berlin;

Prof. Dr. M. Heberer, Basel;

J. Klauber, Berlin

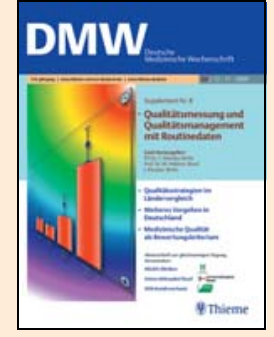

DMW S 8/2009

Qualitätsmessung und Qualitätsmanagement mit Routinedaten

Gast-Herausgeber:

PD Dr. T. Mansky, Berlin;

Prof. Dr. M. Heberer, Basel;

J. Klauber, Berlin 